\title{
CUBIERTAS VEGETALES EN PRODUCCIÓN ORGÁNICA DE FRAMBUESA: EFECTOS SOBRE EL CONTENIDO DE NUTRIENTES DEL SUELO Y EN EL CRECIMIENTO Y PRODUCCIÓN DE LAS PLANTAS
}

\author{
Cover crops in organic raspberry production: effects on soil nutrient content, \\ and raspberry growth and yield
}

\author{
Carlos Ovalle M. ${ }^{1}$ *, María Inés González A. ${ }^{1}$ Alejandro del Pozo L. ${ }^{2}$ Juan Hirzel C. ${ }^{1}$ \\ y Viviana Hernaiz. ${ }^{3}$
}

\section{A B S T R A C T}

A study of the evaluation and selection of cover crops to improve soil management in an organic orchard of raspberry (Rubus idaeus L.) was carried out. The evaluated cover crops were: a mixture of subterranean clover (Trifolium subterraneum L.) and balansa clover (T. michelianum Savi), birdsfoot trefoil (Lotus corniculatus L.), white clover (T. repens L.), fescue (Festuca arundinacea Schreb.), a mixture of white clover and fescue, and a control without vegetation. The trial was conducted in Chillán, Chile ( $36^{\circ} 37^{\prime} \mathrm{S}$, $\left.72^{\circ} 00^{\prime} \mathrm{W}\right)$, during two seasons. The experimental design was randomized blocks with four replicates. Planting distance between raspberry rows was $3 \mathrm{~m}$, and each experimental plot was $60 \mathrm{~m}^{2}$. Covers that included white clover as a single species and white clover in mixture with tall fescue had the highest plant density (828 and 1788 plant $\mathrm{m}^{-2}$, in the second year, respectively), biomass (9.3 and $10.4 \mathrm{t} \mathrm{DM} \mathrm{ha}^{-1} \mathrm{yr}^{-1}$, in the second year, respectively) and specific contribution of the sown species (more than $90 \%$ contribution of sown species). Mixture of annual species did not have a good performance, particularly during the second season. A clear effect of the covers on soil content of nitrogen $(\mathrm{N})$ and potassium $(\mathrm{K})$ was found; however, soil organic matter content and $\mathrm{pH}$ were not affected. Weed growth was best controlled by the cover crops that included fescue and/or white clover.

Key words: Rubus idaeus, Trifolium repens, Trifolium michelianum, Trifolium subterraneum, Lotus corniculatus, Festuca arundinacea.

\author{
R E S U M E N
}

Se realizó un estudio de evaluación y selección de cubiertas vegetales para mejorar el manejo de suelos entre las hileras de un huerto orgánico de frambuesa (Rubus idaeus L.). Las cubiertas evaluadas fueron: mezcla de trébol subterráneo (Trifolium subterraneum L.) con trébol balansa (T. michelianum Savi), lotera (Lotus corniculatus L.), trébol blanco (T. repens L.), festuca (Festuca arundinacea Schreb.), mezcla de trébol blanco y festuca, y un testigo sin cubierta vegetal. El ensayo se realizó en Chillán, Chile ( $36^{\circ} 37^{\prime}$ lat. Sur, $72^{\circ} 00^{\prime}$ long. Oeste), durante dos temporadas. El diseño experimental fue de bloques al azar con cuatro repeticiones. La distancia de plantación entre hileras de frambuesa fue de $3 \mathrm{~m}$ y cada parcela experimental tenía una superficie de $60 \mathrm{~m}^{2}$. Las cubiertas que incluyeron trébol blanco solo o en mezcla con festuca presentaron la mayor densidad de plantas ( 828 y 1.788 plantas $\mathrm{m}^{-2}$ en el segundo año, respectivamente) producción de biomasa (9,3 and10,4 t MS ha ${ }^{-1}$ año-1 $^{-1}$ en el segundo año, respectivamente) y contribución específica de las especies sembradas (sobre $90 \%$ de contribución de las especies sembradas). La mezcla de especies anuales no tuvo un buen comportamiento, particularmente durante la segunda temporada. Se encontró un claro efecto de las cubiertas sobre el contenido de N y K del suelo; sin embargo, no modificaron el contenido de materia orgánica ni $\mathrm{pH}$ del suelo. Las cubiertas que controlaron en mejor forma el crecimiento de las malezas fueron aquellas que incluían festuca y/o trébol blanco.

Palabras clave: Rubus idaeus, Trifolium repens, Trifolium michelianum, Trifolium subterraneum, Lotus corniculatus, Festuca arundinacea.

\footnotetext{
${ }^{1}$ Instituto de Investigaciones Agropecuarias, Centro Regional de Investigación Quilamapu, Casilla 426, Chillán, Chile.

E-mail: covalle@inia.cl*Autor para correspondencia.

${ }^{2}$ Universidad de Talca, Facultad de Ciencias Agrarias, Casilla 747, Talca, Chile.

${ }^{3}$ Universidad Adventista de Chile, Camino Mariposas km 7, Chillán, Chile.

Recibido: 17 de julio de 2006. Aceptado: 4 de diciembre de 2006.
} 


\section{INTRODUCCIÓN}

La frambuesa (Rubus idaeus L.) es en la actualidad un cultivo de gran importancia económica y social en Chile, principalmente porque representa un importante ingreso de divisas para el país y genera una alta demanda de mano de obra estacional. En su modalidad orgánica la exportación de frambuesa presenta excelentes perspectivas de crecimiento debido al sobreprecio obtenido en los mercados, donde se logran diferencias favorables de entre un 30 y $60 \%$ para producto fresco y congelado, respectivamente (INE, 1997). Existen en el país alrededor de 212 hectáreas de berries (frambuesa, arándano y mora) que usan el sistema orgánico de producción (Eguillor, 2004).

En producción orgánica la nutrición nitrogenada de las plantas es un problema de alta relevancia debido a que los fertilizantes convencionales de alta solubilidad se encuentran restringidos en su utilización. Fuentes alternativas de nitrógeno son los composts, los abonos verdes y el cultivo entrehileras de plantas leguminosas fijadoras de $\mathrm{N}$.

El uso de cubiertas vegetales ha sido descrito como una alternativa sustentable de manejo del suelo, que presenta múltiples ventajas, tales como reducir el escurrimiento del agua, evitar la erosión (Frye y Blevins, 1989) y contribuir al mantenimiento y/o mejoramiento de la características físicas del suelo, en especial de la estructura, porosidad, y capacidad de infiltración del agua, evitando o mitigando la compactación del suelo (McVay et al., 1989). Además contribuyen a la disminución de la población de malezas de difícil manejo y permiten el control de algunas especies de nemátodos que dañan los huertos frutales (Aballay e Insunza, 2002).

Las plantas generalmente recomendadas como cubierta vegetal pertenecen a las familias Fabaceae (leguminosas), Brassicaceae (crucíferas) y Poaceae (gramíneas). Las cubiertas de leguminosas tienen la gran ventaja de aportar $\mathrm{N}$ por fijación biológica (Campillo et al., 2003; Ovalle et al., 2006a). Por lo anterior, las gramíneas se siembran casi siempre asociadas con leguminosas, pues permiten obtener una masa de vegetación más importante y además presentan sistemas de raíces complementarios a los de las leguminosas, lo que tiene un efecto favorable sobre la estructura del suelo.
La cantidad de $\mathrm{N}$ aportado por las leguminosas depende de la especie utilizada como cubierta, de la biomasa total producida, del porcentaje de $\mathrm{N}$ en el tejido de la planta, de la capacidad de fijar el $\mathrm{N}$ del aire, y de las condiciones ambientales que afectan el crecimiento de la leguminosa seleccionada (Sullivan, 2003). En praderas sembradas con trébol blanco durante dos temporadas, el primer año se obtuvieron entre 262,7 y $283,4 \mathrm{~kg} \mathrm{ha}^{-1}$ de $\mathrm{N}$ total fijado en la biomasa del trébol, aumentando en la segunda temporada a niveles entre 438 y $442,2 \mathrm{~kg} \mathrm{~N} \mathrm{ha}^{-1}$ (Campillo et al., 2003). En praderas de leguminosas anuales evaluadas bajo condiciones de secano, las tasas de fijación oscilaron entre 15 y $20 \mathrm{~kg} \mathrm{~N} \mathrm{t}^{-1} \mathrm{MS}$ producida (Ovalle et al., 2006a).

Se estima que el $40 \%$ del $N$ contenido en las plantas de una cubierta vegetal puede llegar a estar disponible en el suelo el primer año, mientras que el $60 \%$ restante se encontrará disponible si la cubierta vegetal es incorporada como abono verde (Hoyt, 1987). La mineralización del $\mathrm{N}$ aportado por el abono verde y su utilización por cultivos subsiguientes depende de la composición química de las leguminosas forrajeras, de las propiedades del suelo, del manejo del abono verde (Ladd et al., 1985; Harris y Hesterman, 1990; Varco et al., 1993) y de la sincronización entre las leguminosas que liberan $\mathrm{N}$ y la captación por los cultivos subsiguientes que no son leguminosas (Dou et al., 1994; Stute y Posner, 1995).

Bowen y Freyman (1995) estudiaron en frambuesa la influencia de cubiertas vegetales de leguminosas perennes, trébol blanco (T repens) y ballica perenne (Lolium perenne L.), comparando ambos tratamientos con la mantención de la entrehilera libre de vegetación. Se encontró mayor crecimiento en altura y diámetro de los retoños por efecto de la cubierta de leguminosa. En producción de fruto no se encontraron diferencias entre la cubierta de trébol blanco y el tratamiento sin cubierta, pero el rendimiento con cubierta de trébol blanco fue un $9 \%$ mayor que con cubierta de ballica perenne. De la misma manera, el contenido de $\mathrm{N}$ de las hojas, el área foliar, el peso de hojas y la tasa de asimilación neta de $\mathrm{CO}_{2}$, fueron mayores en las plantas que crecieron bajo la influencia de cubiertas de trébol blanco entre las hileras, que aquellas en que la cubierta fue de ballica perenne.

En relación con el control de malezas, se han empleado con éxito algunos cultivos anuales como la 
avena (Avena sativa), ballica italiana (Lolium multiflorum Lam.), centeno (Secale cereale L.), tréboles (Trifolium spp.), y algunas especies perennes como festuca y ballica perenne. Incluso se ha recomendado el uso de mezclas botánicas con diferentes grados de floración para ayudar a los enemigos naturales de plagas de frutales, pues así cuentan con una disponibilidad prolongada de néctar para su alimentación (Ormeño, 2002). Los mejores resultados con cubiertas vegetales se han logrado en especies frutales con receso invernal (pomáceas, carozos, vides). En este caso, las praderas de crecimiento invernal que se desarrollan entre las hileras, no interfieren con el crecimiento activo de primaveraverano de los árboles y, además de mejorar las condiciones físicas del suelo y su contenido de materia orgánica, sirven para controlar malezas invernales anuales que crecen entre las hileras de estos frutales (Ormeño, 2002).

El objetivo del estudio fue seleccionar especies y/o mezclas de especies pratenses de leguminosas y gramíneas, factibles de ser utilizadas como cultivo entre las hileras de frambuesa en huertos orgánicos. Además de estudiar el efecto de las cubiertas vegetales sobre el crecimiento y la producción de las plantas de frambuesa, se evaluará su efecto sobre el contenido de nutrientes del suelo.

\section{MATERIALES Y MÉTODOS}

El ensayo se realizó en el fundo "Los Guindos" $\left(36^{\circ} 37^{\prime}\right.$ lat. Sur, $72^{\circ} 00^{\prime}$ long. Oeste), ubicado en el sector "Las Mariposas", comuna de Chillán, provincia de Ñuble, Región del Bío-Bío. El clima es mediterráneo húmedo con $1.272 \mathrm{~mm}$ de precipitación media anual, concentrados entre los meses de abril y octubre. La temperatura media anual es de $13,9^{\circ} \mathrm{C}$, la mínima de julio de $3,0^{\circ} \mathrm{C}$, la máxima de enero de $28,6^{\circ} \mathrm{C}$, y la suma térmica promedio es de $2.948^{\circ} \mathrm{C}$-día. El período libre de heladas es de cinco a seis meses, y el largo de la estación de crecimiento entre ocho y nueve meses (del Pozo y del Canto, 1999). El suelo pertenece a la serie Arrayán (typic Melanoxerands) formado sobre cenizas volcánicas, materiales fluviales y materiales fluvio glaciales, compactados y lentamente permeables. Son suelos profundos, de textura media, franco limosos, bien estructurados en superficie (CIREN CORFO, 1999). La densidad aparente es de $0,8 \mathrm{~g} \mathrm{~cm}^{-3}$, presentan un $23 \%$ de retención de agua en los primeros $30 \mathrm{~cm} \mathrm{y}$ $552 \mathrm{~m}^{3} \mathrm{ha}^{-1}$ de agua aprovechable; el $\mathrm{pH}$ varía entre 5,8 y 6,2 y tienen un alto contenido de materia orgánica (del Pozo y del Canto, 1999).

Los tratamientos fueron cinco cubiertas vegetales de diferentes especies forrajeras: trébol balanza + trébol subterráneo; lotera; trébol blanco; festuca; y trébol blanco + festuca, además de un testigo sin cubierta vegetal. En el Cuadro 1 se indican los tratamientos, cultivares y dosis de semilla.

El experimento se estableció en parcelas de $6 \times 10$ $\mathrm{m}\left(60 \mathrm{~m}^{2}\right)$ en un huerto de frambuesa variedad Heritage de 5 años de edad. La distancia entre hileras de frambuesa era de $3 \mathrm{~m}$ y el número de tallos (cañas) por metro lineal, al inicio del experimento, fue en promedio 12 .

\section{Establecimiento y manejo del experimento}

La preparación del suelo se hizo con dos pasadas de rastra de discos, luego una labor con vibrocultivador y rastrillo manual. La siembra se realizó con una máquina sembradora manual (Planet Junior, Allen

Cuadro 1. Ensayo de cubiertas vegetales. Identificación de los tratamientos, especies, cultivares y dosis de semillas. Table 1. Cover crop experiment. Treatments identification, species, cultivars, and seed rate.

\begin{tabular}{clllr}
\hline $\mathbf{N}^{\mathbf{0}}$ & Tratamientos de cubiertas & Especie & Cultivar & $\begin{array}{c}\text { Dosis semilla } \\
\left(\mathbf{k g ~ h a}^{-1} \mathbf{)}\right.\end{array}$ \\
\hline 1 & Manejo tradic., sin cubierta vegetal & -- & - & - \\
2 & Cubierta de leguminosas anuales & Trébol balansa & Paradana & 5 \\
& & Trébol subterráneo & Denmark & 10 \\
& & & Antas & 10 \\
3 & Cubierta de leguminosa perenne & Lotera & San Gabriel & 6 \\
4 & Cubierta de leguminosa perenne & Trébol blanco & Will & 5 \\
5 & Cubierta de gramínea perenne & Festuca & Manade & 10 \\
6 & Cubierta de leguminosa perenne + & Trébol blanco & Will & 5 \\
& gramínea perenne & Festuca & Manade & 10 \\
\hline
\end{tabular}


Co., Philadelphia, Pennsylvania, EE.UU.) el 1 de julio de 2003, en hileras separadas a $20 \mathrm{~cm}$ y dispuestas en el mismo sentido de la plantación de frambuesa. La fertilización para el establecimiento consistió en la aplicación al voleo y posterior incorporación de $150 \mathrm{~kg}$ de $\mathrm{P}_{2} \mathrm{O}_{5}$ como roca fosfórica; $66 \mathrm{~kg}$ de Ca y $36 \mathrm{~kg}$ de $\mathrm{S}$ como Fertiyeso; 110 $\mathrm{kg}$ de $\mathrm{K}, 90 \mathrm{~kg}$ de $\mathrm{Mg}$ y $110 \mathrm{~kg}$ de $\mathrm{S}$ como Sulpomag; y $2 \mathrm{~kg}$ de B como boronatrocalcita. En forma adicional se aplicaron otros $110 \mathrm{~kg}$ de K, 90 $\mathrm{kg}$ de $\mathrm{Mg}$ y $110 \mathrm{~kg}$ de S como Sulpomag sobre la línea de siembra.

\section{Evaluaciones en la cubierta vegetal}

La densidad de plantas se evaluó mediante muestras obtenidas con tubos de policloruro de vinilo (PVC) de $78 \mathrm{~cm}$ de diámetro, que se introdujeron en el suelo al azar en seis sitios de cada parcela, y posteriormente se realizó el recuento de plántulas. Éstas se separaron por especie, según el tratamiento, y se contabilizaron. Este muestreo se hizo en dos oportunidades: fines de julio de 2003 y mediados de septiembre de 2004. La producción de fitomasa y composición botánica se evaluó mediante el corte de cuatro cuadrantes de $0,5 \mathrm{~m}^{2}$ por parcela. El corte de la fitomasa se realizó a $5 \mathrm{~cm}$ del suelo abarcando la totalidad de la biomasa aérea circunscrita en el cuadrante. Las muestras se secaron en horno con ventilación forzada a $65^{\circ} \mathrm{C}$ por $72 \mathrm{~h}$. Se obtuvo el peso de la materia seca total, y posteriormente en laboratorio se determinó la composición botánica, separando manualmente las especies sembradas de las malezas dentro de cada mezcla. Este procedimiento se realizó en cortes sucesivos realizados cada 28 días, de acuerdo al crecimiento de la cubierta vegetal.

\section{Evaluaciones en las plantas de frambuesa}

En abril de 2004 se contó el número de laterales por caña en 10 cañas de cada parcela, y en septiembre del mismo año se contó el número de cañas en $2 \mathrm{~m}$ lineales de cada parcela. La cosecha se realizó sobre $2 \mathrm{~m}$ lineales marcados previamente en cada tratamiento. En la primera temporada se cosechó entre el 12 de diciembre de 2003 y el 13 de marzo de 2004, y en la segunda temporada se cosechó entre el 22 de diciembre de 2004 y el 3 de marzo de 2005. La única determinación de cosecha fue el peso total de la fruta.

\section{Evaluaciones en el suelo}

Para conocer la evolución de las variables químicas del suelo, materia orgánica, pH, N, P y K, se realizó un seguimiento en el tiempo en tres tratamientos: sin cubierta vegetal, cubierta de trébol blanco solo, y cubierta de trébol blanco más festuca. Para este efecto se empleó una modificación del método de incubación de suelos in situ propuesto por Raison et al. (1987). Al inicio del experimento se instalaron tubos de PVC en el suelo, cuyas dimensiones fueron $25 \mathrm{~cm}$ de largo y $10 \mathrm{~cm}$ de diámetro. El número de tubos por unidad experimental fue equivalente al número de muestreos que se realizaron (6) durante la temporada.

Se dispuso en los tubos la biomasa aérea proveniente de cada corte de la cubierta vegetal. Para ello, se calculó la disponibilidad de materia seca de la pradera, y se dispuso en cada tubo la cantidad de fitomasa proporcional al área del tubo $\left(74,6 \mathrm{~cm}^{2}\right)$. Periódicamente, cada 30 a 45 días, se colectó un tubo por unidad experimental, y las muestras de suelo correspondientes se sometieron a análisis de MO (Walkey-Black modificado, oxidación con $\mathrm{K}_{2} \mathrm{Cr}_{2} \mathrm{O}_{7}$ y determinación por colorimetría), $\mathrm{N}$ mineral $(\mathrm{KCl} 2 \mathrm{~N}$ $1: 10$ y lectura en un autoanalizador a inyección de flujo segmentado) (SKALAR, SA 4000, Breda, The Netherlands), P extractable Olsen (bicarbonato de sodio $0,5 \mathrm{M}$ a $\mathrm{pH} 8,5$ y determinación por colorimetría) y $\mathrm{K}$ intercambiable (extracción con solución de acetato de amonio 1 mol L-1, pH 7,0 y espectrofotometría de absorción y emisión atómica).

Para el análisis de tejidos se determinó la materia seca, a través de secado en estufa a $65^{\circ} \mathrm{C}$ durante $48 \mathrm{~h}$; el contenido de nitrógeno por digestión Kjeldhal y determinación por destilación y titulación automática; el contenido de fósforo por digestión ácida con $\mathrm{HCl} 2 \mathrm{M}$ de las cenizas obtenidas por calcinación de la muestra a $500{ }^{\circ} \mathrm{C}$ y determinación por colorimetría del fosfo-vanado-molíbdico; contenido de potasio por digestión ácida con $\mathrm{HCl} 2 \mathrm{M}$ de las cenizas obtenidas por calcinación de las muestras a $500{ }^{\circ} \mathrm{C}$ y determinación por espectrofotometría de llama por emisión atómica.

\section{Diseño experimental y análisis estadístico}

El diseño fue bloques completos al azar con cuatro repeticiones. Se realizó análisis de varianza y se utilizó la prueba de rango múltiple de Duncan $(\mathrm{P} \leq 0,05)$ para la comparación de medias. 


\section{RESULTADOS Y DISCUSIÓN}

\section{Variables evaluadas en la cubierta vegetal}

El primer año se observó una población de plantas significativamente mayor $(P \leq 0,05)$ en la mezcla de festuca y trébol blanco seguida de la mezcla de leguminosas anuales, trébol balansa y trébol subterráneo (Cuadro 2). Las cubiertas de una sola especie tuvieron una menor densidad de plantas, producto de la menor dosis de semilla con que fueron sembradas (Cuadro 1). En el segundo año de la pradera, el tratamiento con mayor densidad poblacional fue nuevamente la mezcla de festuca y trébol blanco, confirmando lo observado en la temporada anterior.

La ausencia de plantas en la mezcla de trébol subterráneo y trébol balansa en la segunda temporada (Cuadro 2) se debió a que el huerto de frambuesa fue regado gravitacionalmente durante todo el período estival, lo que provocó, por una parte, una proliferación de malezas de crecimiento estival y, por otra, que las semillas de estas leguminosas germinaran anticipadamente, y en el otoño siguiente el banco de semillas del suelo no disponía de semillas blandas para la germinación de la segunda temporada. Este comportamiento de las leguminosas anuales al ser sometidas a riego estival es similar al fenómeno conocido como "falsas partidas" (del Pozo et al., 2000), que ocurre en praderas de secano cuando se presentan lluvias de verano-otoño. En este caso también se produjo una alta mortalidad de plantas y agotamiento del banco de semillas.

Cuadro 2. Densidad de plantas $\left(\mathrm{N}^{\circ} \mathrm{m}^{-2}\right)$ de diferentes cubiertas vegetales sembradas entre las hileras de un huerto orgánico de frambuesa en julio 2003 (año de establecimiento) y en septiembre del 2004.

Table 2. Plant density (Number $\mathrm{m}^{-2}$ ) of different cover crops between rows in an organic raspberry orchard in July 2003 (year of establishment) and in September 2004.

\begin{tabular}{lcc}
\hline Tratamientos & $\mathbf{2 0 0 3 - 2 0 0 4}$ & $\mathbf{2 0 0 4 - 2 0 0 5}$ \\
\hline Trébol balansa + & & \\
$\quad$ trébol subterráneo & $343 \mathrm{~b}$ & $0 \mathrm{c}$ \\
Lotera & $122 \mathrm{~d}$ & $350 \mathrm{bc}$ \\
Trébol blanco & $236 \mathrm{c}$ & $828 \mathrm{~b}$ \\
Festuca & $140 \mathrm{~d}$ & $701 \mathrm{~b}$ \\
Festuca + trébol blanco & $451 \mathrm{a}$ & $1.799 \mathrm{a}$ \\
\hline
\end{tabular}

Medias seguidas por letras distintas en una misma columna difieren significativamente según Prueba de Duncan $(\mathrm{P} \leq 0,05)$.
Además, durante la segunda temporada se observó proliferación de trébol blanco, tanto en la mezcla de leguminosas anuales como en las cubiertas de lotera y de festuca solas. Este fenómeno se puede explicar por la alta población de trébol blanco naturalizado que existe en el suelo donde se realizó el ensayo, y la alta respuesta de esta especie a la aplicación de fertilizantes fosfatados, perjudicando el establecimiento de otras leguminosas como la lotera, el trébol balansa y el trébol subterráneo.

La mayor producción de biomasa $(\mathrm{P} \leq 0,05)$ de las especies sembradas durante la temporada 20032004 se obtuvo en las mezclas de trébol balansa con trébol subterráneo, y de festuca con trébol blanco; los tratamientos con una sola especie presentaron una producción inferior sin diferencias estadísticas (Cuadro 3). En la temporada 20042005 se observó una mayor producción de la mezcla festuca + trébol blanco, con una producción superior a $9 \mathrm{t} \mathrm{MS} \mathrm{ha}^{-1}$, siendo similar $(\mathrm{P} \leq 0,05)$ a la cubierta de trébol blanco solo (Cuadro 3). Asimismo, se confirmó la presencia de trébol blanco espontáneo en todos los tratamientos, lo que igualó las producciones de fitomasa total. La contribución específica de las especies sembradas aumentó en el tiempo en las cubiertas de trébol blanco solo, festuca sola y la mezcla de ambas especies (Cuadro 4). Destaca el hecho que las cubiertas con trébol blanco presentaron una contribución de un 98\% de la especie sembrada en la primavera de la segunda temporada de evaluación, desplazando a las especies espontáneas o malezas a niveles inferiores a $2 \%$. Por otra parte, en las cubiertas de leguminosas anuales y de lotera predominaron claramente las especies no sembradas, dominando el trébol blanco espontáneo.

No se observaron diferencias significativas $(\mathrm{P} \geq$ 0,05 ) en el contenido de $\mathrm{N}$ de la biomasa entre los tratamientos que incluían una leguminosa (Cuadro 5). La invasión de trébol blanco espontáneo en los tratamientos que no lo incluían inicialmente, explica esta homogeneidad en el resultado. A pesar de esta situación, el contenido de $\mathrm{N}$ de la cubierta de festuca sola fue significativamente menor $(\mathrm{P} \leq$ 0,05). El mismo patrón se observó en el contenido de P. Respecto al contenido de K no se observaron diferencias $(P \geq 0,05)$ entre las cubiertas vegetales ensayadas. 
Cuadro 3. Fitomasa total y de las especies sembradas como cubiertas vegetales en un huerto orgánico de frambuesas en dos temporadas de crecimiento.

Table 3. Total biomass and biomass from sown species as cover crops in an organic raspberry orchard in two growing seasons.

\begin{tabular}{|c|c|c|c|c|}
\hline \multirow[t]{2}{*}{ Tratamientos } & \multicolumn{2}{|c|}{ 2003-2004 } & \multicolumn{2}{|c|}{ 2004-2005 } \\
\hline & $\begin{array}{c}\text { Total } \\
\left(\text { t ha }^{-1}\right)\end{array}$ & $\begin{array}{c}\text { Especie } \\
\text { sembrada } \\
\left(\mathrm{t} \mathrm{ha}^{-1}\right)\end{array}$ & $\begin{array}{c}\text { Total } \\
\left(\text { t ha }^{-1}\right)\end{array}$ & $\begin{array}{c}\text { Especie } \\
\text { sembrada } \\
\left(\mathrm{t} \mathrm{ha}^{-1}\right)\end{array}$ \\
\hline Trébol balansa + trébol subterráneo & $9,256 \mathrm{a}$ & $4,540 \mathrm{a}$ & 9,647 a & $0,338 \mathrm{c}$ \\
\hline Lotera & $7,037 \mathrm{bc}$ & $0,820 \mathrm{~b}$ & $11,809 \mathrm{a}$ & $1,025 \mathrm{c}$ \\
\hline Trébol blanco & $6,229 \mathrm{c}$ & $1,958 \mathrm{~b}$ & $9,334 \mathrm{a}$ & 8,672 a \\
\hline Festuca & $6,528 \mathrm{c}$ & $1,301 \mathrm{~b}$ & 9,669 a & $6,048 \mathrm{~b}$ \\
\hline Festuca + trébol blanco & $7,638 \mathrm{~b}$ & $3,622 \mathrm{a}$ & $10,491 \mathrm{a}$ & $9,420 \mathrm{a}$ \\
\hline
\end{tabular}

Medias seguidas por letras distintas en una misma columna difieren significativamente según Prueba de Duncan $(\mathrm{P} \leq 0,05)$.

Cuadro 4. Composición botánica (\% de especie sembrada) de las cubiertas vegetales establecidas en un huerto orgánico de frambuesa.

Table 4. Botanical composition (\% of sown species) of cover crops established in an organic raspberry orchard.

\begin{tabular}{lccc}
\hline Tratamientos & Noviembre 2003 & Julio 2004 & Noviembre 2004 \\
\hline Trébol balansa + trébol subterráneo & $61 \mathrm{a}$ & $0 \mathrm{c}$ & $12 \mathrm{c}$ \\
Lotera & $3 \mathrm{c}$ & $11 \mathrm{c}$ & $1 \mathrm{c}$ \\
Trébol blanco & $23 \mathrm{~b}$ & $87 \mathrm{a}$ & $98 \mathrm{a}$ \\
Festuca & $4 \mathrm{c}$ & $61 \mathrm{~b}$ & $52 \mathrm{~b}$ \\
Festuca + trébol blanco & $39 \mathrm{ab}$ & $98 \mathrm{a}$ & $98 \mathrm{a}$ \\
\hline
\end{tabular}

Medias seguidas por letras distintas en una misma columna difieren significativamente según Prueba de Duncan $(\mathrm{P} \leq 0,05)$

Cuadro 5. Concentración de nitrógeno (N), fósforo (P) y potasio (K) en praderas sembradas como cubiertas vegetales en un huerto orgánico de frambuesa (noviembre 2004).

Table 5. Concentration of nitrogen $(N)$, phosphorus $(P)$ and potassium $(K)$ in pastures sown as cover crops in an organic raspberry orchard (November 2004).

\begin{tabular}{lccc}
\hline Tratamientos & $\mathbf{N}$ & $\mathbf{P}$ & $\mathbf{K}$ \\
\cline { 2 - 4 } & & $\mathbf{\%}$ & \\
\hline Trébol balansa + trébol subterráneo & $3,82 \mathrm{a}$ & $0,38 \mathrm{a}$ & $2,63 \mathrm{a}$ \\
Lotera & $4,03 \mathrm{a}$ & $0,37 \mathrm{a}$ & $2,70 \mathrm{a}$ \\
Trébol blanco & $4,49 \mathrm{a}$ & $0,40 \mathrm{a}$ & $2,93 \mathrm{a}$ \\
Festuca & $2,61 \mathrm{c}$ & $0,26 \mathrm{~b}$ & $2,42 \mathrm{a}$ \\
Festuca + trébol blanco & $4,24 \mathrm{a}$ & $0,38 \mathrm{a}$ & $2,06 \mathrm{a}$ \\
\hline Medias seguidas por letras distintas en una misma columna difieren significativamente según Prueba de
\end{tabular}

Variables evaluadas en la planta de frambuesa

No se observaron diferencias significativas en el número de laterales por caña y el número de cañas por metro lineal $(\mathrm{P}>0,05)$ en la distintas cubiertas vegetales (Cuadro 6). También la producción de frutos fue similar $(\mathrm{P}>0,05)$ entre tratamientos durante ambas temporadas (Figura 1).
Evolución del contenido de nutrientes del suelo El pH y el contenido de MO del suelo no se vieron influenciados por las cubiertas vegetales entre las hileras de frambuesa. Este resultado se debió a que el experimento sólo se evaluó dos temporadas y el suelo, como se puede apreciar en el tratamiento testigo sin cubierta, tenía un alto contenido de MO inicial (Cuadro 7). 
Cuadro 6. Número de cañas por metro lineal y número de laterales por caña, en respuesta a diferentes cubiertas vegetales en un huerto orgánico de frambuesa.

Table 6. Number of canes per lineal meter, and number of laterals per cane in response to different cover crops in an organic raspberry orchard.

\begin{tabular}{lcc}
\hline Tratamientos & $\begin{array}{c}\text { Cañas } \\
\left(\mathbf{N}^{\circ} \mathbf{m}^{-1}\right)\end{array}$ & $\begin{array}{c}\text { Laterales } \\
\left(\mathbf{N}^{\circ} \mathbf{c a n ̃}^{-1}\right)\end{array}$ \\
\hline $\begin{array}{l}\text { Sin cubierta vegetal } \\
\text { Trébol balansa }+\end{array}$ & $62 \mathrm{a}$ & $18 \mathrm{a}$ \\
$\quad$ trébol subterráneo & $72 \mathrm{a}$ & $17 \mathrm{a}$ \\
Lotera & $70 \mathrm{a}$ & $18 \mathrm{a}$ \\
Trébol blanco & $61 \mathrm{a}$ & $18 \mathrm{a}$ \\
Festuca & $62 \mathrm{a}$ & $17 \mathrm{a}$ \\
Festuca + trébol blanco & $62 \mathrm{a}$ & $18 \mathrm{a}$ \\
\hline
\end{tabular}

Medias seguidas porigual letra en las columnas no presentan diferencias significativas entre sí según Prueba de Duncan $(\mathrm{P} \leq 0,05)$.
El contenido de $\mathrm{N}$ mineral no presentó diferencias entre tratamientos en el período de julio a octubre el primer año y de abril a junio el segundo año, cuando la tasa de mineralización podría ser más baja por condiciones de temperatura (Mengel, 1996; Tisdale et al., 1999; He et al., 2000). Sin embargo, entre los meses de noviembre y enero se detectaron diferencias, con un contenido de $\mathrm{N}$ significativamente mayor $(\mathrm{P} \leq 0,05)$ en los tratamientos con trébol blanco, ya sea solo o con festuca. Este comportamiento se explica por la activa mineralización en este período, favorecida por las condiciones ambientales del suelo. Una situación similar fue descrita por He et al. (2000) y por Eghball (2000) para aplicaciones de enmiendas orgánicas al suelo.

El contenido de $\mathrm{P}$ fue inusualmente alto en todos los tratamientos, producto de la historia de fertilización
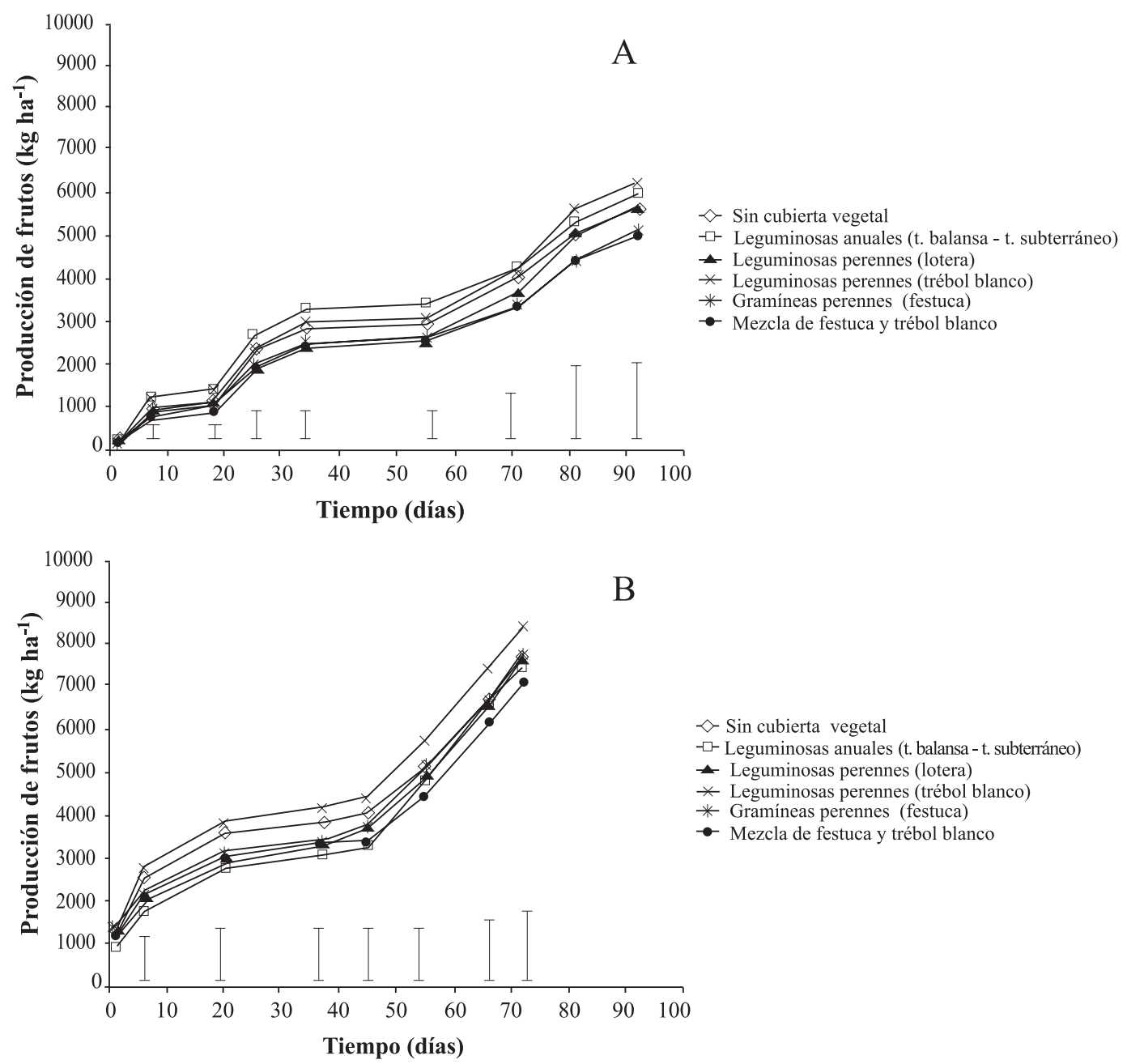

$\prec$ Sin cubierta vegetal

$\rightarrow \square$ Leguminosas anuales (t. balansa - t. subterráneo)

- Leguminosas perennes (lotera)

* Leguminosas perennes (trébol blanco)

* Gramíneas perennes (festuca)

- Mezcla de festuca y trébol blanco

Figura 1. Producción acumulada de frutos de frambuesa $\left(\mathrm{kg} \mathrm{ha}^{-1}\right)$ con relación a la cubierta entrehileras. A) Temporada 2004; B) Temporada 2005.

Figure 1. Cumulative fruit production of raspberry $\left(\mathrm{kg} \mathrm{ha}^{-1}\right)$ in relation to the cover crop. A) 2004 season; B) 2005 season. 
Cuadro 7. Variación en el tiempo del pH y del contenido de materia orgánica, N, P y K en el suelo, bajo diferentes cubiertas vegetales en un huerto orgánico de frambuesa durante la temporada 2004-2005.

Table 7. Variation over time of $\mathrm{pH}$, and soil content of organic matter, $\mathrm{N}$, $\mathrm{P}$, and $\mathrm{K}$ under different cover crops in an organic raspberry orchard during the 2004-2005 growing season.

\begin{tabular}{lcrrrrr}
\hline Tratamientos & $\mathbf{0 7 / 0 7 / 2 0 0 4}$ & $\mathbf{2 0 / 1 0 / 2 0 0 4}$ & $\mathbf{2 6 / 1 1 / 2 0 0 4}$ & $\mathbf{0 6 / 0 1 / 2 0 0 5}$ & $\mathbf{0 6 / 0 4 / 2 0 0 5}$ & $\mathbf{0 6 / 0 6 / 2 0 0 5}$ \\
\hline \multicolumn{7}{c}{ Materia orgánica, \% } \\
Sin cubierta & $8,95 \mathrm{a}$ & $9,22 \mathrm{a}$ & $10,01 \mathrm{a}$ & $9,99 \mathrm{a}$ & $8,88 \mathrm{a}$ & $8,69 \mathrm{a}$ \\
Trébol blanco & $9,26 \mathrm{a}$ & $8,89 \mathrm{a}$ & $9,21 \mathrm{a}$ & $10,11 \mathrm{a}$ & $9,54 \mathrm{a}$ & $9,11 \mathrm{a}$ \\
T. blanco + festuca & $9,25 \mathrm{a}$ & $8,35 \mathrm{a}$ & $8,94 \mathrm{a}$ & $9,77 \mathrm{a}$ & $8,16 \mathrm{a}$ & $9,25 \mathrm{a}$
\end{tabular}

\begin{tabular}{lrrrrrr}
\multicolumn{1}{c}{$\mathrm{pH}$ agua } & & & & \\
Sin cubierta & $7,01 \mathrm{a}$ & $6,61 \mathrm{a}$ & $6,70 \mathrm{a}$ & $6,63 \mathrm{a}$ & $6,80 \mathrm{a}$ & $6,93 \mathrm{a}$ \\
Trébol blanco & $7,07 \mathrm{a}$ & $6,76 \mathrm{a}$ & $6,49 \mathrm{a}$ & $6,35 \mathrm{a}$ & $6,87 \mathrm{a}$ & $6,95 \mathrm{a}$ \\
T. blanco + festuca & $7,13 \mathrm{a}$ & $6,87 \mathrm{a}$ & $6,69 \mathrm{a}$ & $6,31 \mathrm{a}$ & $6,77 \mathrm{a}$ & $6,97 \mathrm{a}$
\end{tabular}

\begin{tabular}{lcrrrrr}
\multicolumn{7}{c}{${\mathrm{N}-\mathrm{NO}_{3}^{-}+\mathrm{N}^{-} \mathrm{NH}_{4}, \mathrm{mg} \mathrm{kg}^{-1}}$} \\
Sin cubierta & $7,03 \mathrm{a}$ & $40,97 \mathrm{a}$ & $50,49 \mathrm{~b}$ & $72,69 \mathrm{~b}$ & $10,84 \mathrm{a}$ & $8,35 \mathrm{~b}$ \\
Trébol blanco & $7,96 \mathrm{a}$ & $35,84 \mathrm{a}$ & $113,41 \mathrm{a}$ & $240,98 \mathrm{a}$ & $13,61 \mathrm{a}$ & $20,59 \mathrm{a}$ \\
T. blanco + festuca & $5,95 \mathrm{a}$ & $22,94 \mathrm{a}$ & $50,27 \mathrm{~b}$ & $276,90 \mathrm{a}$ & $21,77 \mathrm{a}$ & $9,60 \mathrm{~b}$
\end{tabular}

\begin{tabular}{lcrrrrr}
\multicolumn{7}{c}{ P Olsen, $\mathrm{mg} \mathrm{kg}^{-1}$} \\
Sin cubierta & $94,62 \mathrm{a}$ & $103,08 \mathrm{a}$ & $122,51 \mathrm{a}$ & $90,10 \mathrm{a}$ & $82,88 \mathrm{a}$ & $92,91 \mathrm{a}$ \\
Trébol blanco & $91,76 \mathrm{a}$ & $106,56 \mathrm{a}$ & $131,99 \mathrm{a}$ & $112,14 \mathrm{a}$ & $96,96 \mathrm{a}$ & $104,69 \mathrm{a}$ \\
T. blanco + festuca & $82,35 \mathrm{a}$ & $111,45 \mathrm{a}$ & $113,41 \mathrm{a}$ & $105,99 \mathrm{a}$ & $88,24 \mathrm{a}$ & $98,97 \mathrm{a}$
\end{tabular}

\begin{tabular}{lrrrrrr} 
& $\mathrm{K}, \mathrm{mg} \mathrm{kg}^{-1}$ & & & & \\
Sin cubierta & $439,17 \mathrm{a}$ & $446,78 \mathrm{a}$ & $546,42 \mathrm{~b}$ & $348,77 \mathrm{~b}$ & $376,69 \mathrm{~b}$ & $380,05 \mathrm{~b}$ \\
Trébol blanco & $473,45 \mathrm{a}$ & $381,94 \mathrm{a}$ & $828,72 \mathrm{a}$ & $1069,48 \mathrm{a}$ & $514,29 \mathrm{a}$ & $769,19 \mathrm{a}$ \\
T. blanco + festuca & $386,35 \mathrm{a}$ & $416,47 \mathrm{a}$ & $596,90 \mathrm{~b}$ & $980,92 \mathrm{a}$ & $414,12 \mathrm{~b}$ & $621,89 \mathrm{a}$ \\
\hline
\end{tabular}

Medias seguidas por igual letra en las columnas no presentan diferencias significativas según Prueba de Duncan $(\mathrm{P} \leq 0,05)$.

previa del huerto en base a compost y a la elevada aplicación de roca fosfórica $\left(500 \mathrm{~kg} \mathrm{ha}^{-1}\right.$, equivalentes a $150 \mathrm{~kg} \mathrm{P}_{2} \mathrm{O}_{5} \mathrm{ha}^{-1}$ ) al inicio del experimento. En ese contexto, las cubiertas vegetales no mostrarían un claro efecto en términos de incrementar el contenido de $\mathrm{P}$ en el suelo. Respecto del K, se observó también un significativo aumento en el período de mayor mineralización de la MO, puesto que este elemento no forma compuestos orgánicos estables dentro de la planta, siendo liberado rápidamente cuando el tejido vegetal comienza a mineralizarse, lo cual coincide con lo señalado por He et al. (2000).

En síntesis, la incorporación de cubiertas vegetales entre las hileras de un huerto de frambuesa orgánica presentó ventajas al ser comparada con un suelo manejado libre de vegetación. Las cubiertas que experimentaron el mejor comportamiento fueron las que incluyeron trébol blanco, y produjeron altas cantidades de biomasa, comparables con la producción de praderas destinadas a la producción animal en la misma área (Acuña, 1995) y con altos conte- nidos de $\mathrm{N}$ (sobre 3,3\%). Parte de este $\mathrm{N}$ proviene de la reserva del suelo, pero la fracción más importante provendría de la fijación biológica. En efecto, en suelos volcánicos la fijación en trébol blanco ha sido estimada sobre el $90 \%$ del $\mathrm{N}$ contenido en la biomasa, lo que equivale a niveles de fijación sobre $260 \mathrm{~kg} \mathrm{~N} \mathrm{ha}^{-1}$ año $^{-1}$ (Campillo et al., 2003).

El efecto de las cubiertas vegetales sobre la planta de frambuesa, medido al segundo año del establecimiento de las cubiertas, no fue detrimental sobre el crecimiento de las plantas ni sobre su producción. La incorporación de $\mathrm{N}$ por fijación biológica de las leguminosas de la entrehilera permitió incrementar el contenido de $\mathrm{N}$ disponible en el suelo. No obstante, el efecto de las cubiertas sobre los otros macronutrientes estudiados no fue significativo. A este respecto, en un estudio paralelo (Ovalle et al., 2006b), mediante métodos isotópicos con aplicación de $\mathrm{N}^{15}$, se determinó que la transferencia de $\mathrm{N}$ desde la cubierta vegetal de leguminosas herbáceas hacia la planta de frambuesa es efectiva y se verifi- 
có principalmente en hojas y tallos, donde en promedio entre un 18 y un $22 \%$ del $\mathrm{N}$ contenido en la biomasa de la frambuesa provenía de la leguminosa, siendo máximo en hojas en el período de crecimiento más activo de la planta. En los frutos la recuperación detectada fue menor, y no se detectó transferencia de $\mathrm{N}$ hacia las raíces en evaluaciones realizadas al final del ciclo de crecimiento de la planta (Ovalle et al., 2006b). Estudios similares, pero utilizando metodología de abundancia natural con $\mathrm{N}^{15}$, han puesto en evidencia este fenómeno en plantaciones frutales en condiciones de clima tropical (Snoeck et al., 2000).

Se determinó que las cubiertas ejercieron un efecto detrimental sobre la población de las especies espontáneas o malezas que crecen en la entrehilera del huerto. Este efecto fue particularmente notorio en la cubierta de trébol blanco con festuca y trébol blanco solo, debido a la alta población de especies sembradas y a la alta producción de biomasa, lo que permitió un menor desarrollo de las especies espontáneas.

\section{CONCLUSIONES}

1.- Las praderas factibles de ser utilizadas como cubiertas entrehileras en huertos de frambuesa orgánica en suelos trumao del valle central de riego son las de trébol blanco solo y la mezcla de trébol blanco + festuca. Estas praderas presentaron altos valores de densidad de plantas, producción de fitomasa, contribución específica de las especies sembradas y contenido de $\mathrm{N}$ en la biomasa. Las especies de leguminosas anuales no presentaron un buen comportamiento bajo las condiciones de suelos del presente estudio.

2.- Las cubiertas vegetales de trébol blanco solo y trébol blanco + festuca no modificaron el $\mathrm{pH}$ del suelo ni el contenido de MO. El contenido de $\mathrm{N}$ del suelo aumentó en los meses en que las condiciones ambientales edáficas permitieron una alta mineralización de la MO. Similar comportamiento presentó el contenido de K. Las cubiertas entrehilera no modificaron el contenido de $\mathrm{P}$ del suelo.

3.- Las cubiertas vegetales no tuvieron un efecto detrimental sobre el crecimiento ni sobre la producción de las plantas de frambuesa.

4.- Las cubiertas que experimentaron el mejor comportamiento en relación con el control de malezas en la entrehilera del huerto, fueron la mezcla de trébol blanco + festuca, y la cubierta de trébol blanco solo.

\section{LITERATURA CITADA}

Aballay, E., y V. Insunza. 2002. Evaluación de plantas con propiedades nematicidas en el control de Xiphinema index en vid de mesa cv. Thompson Seedless en la zona central de Chile. Agric. Téc. (Chile) 62:357-365.

Acuña, H. 1995. Efecto de la aplicación de fósforo y potasio en la productividad de una pradera de trébol blanco/festuca en un suelo arcilloso. Agric. Téc. (Chile) 55:127-133.

Bowen, P., and S. Freyman. 1995. Ground covers affect raspberry yield, photosynthesis, and nitrogen nutrition of primocanes. HortScience 30:238-241.

Campillo, R., S. Urquiaga, I. Pino, y A. Montenegro. 2003. Estimación de la fijación biológica de nitrógeno en leguminosas forrajeras mediante la metodología del $N^{15}$. Agric. Téc. (Chile) 63:169-179.

CIREN CORFO. 1999. Descripción de suelos y materiales y símbolos: Estudio agrológico VIII Región. p. 526-550. Publicación CIREN 121. Centro de Información de Recursos Naturales (CIREN), Santiago, Chile.
Del Pozo, A., y P. del Canto. 1999. Características del Valle Central. Áreas agroclimáticas y sistemas productivos en las VII y VIII Regiones. 115 p. Instituto de Investigaciones Agropecuarias, Centro Regional de Investigación Quilamapu, Chillán, Chile.

Del Pozo, A., C. Ovalle, J. Aronson, and J. Avendaño. 2000. Developmental responses to temperature and photoperiod in ecotypes of Medicago polymorpha $\mathrm{L}$. along an environmental gradient in Central Chile. Ann. Bot. 85:809-814.

Dou, Z., R.H. Fox, and J.D. Toth. 1994. Tillage effect on seasonal nitrogen availability in corn supplied with legume green manures. Plant Soil 162:203-210.

Eghball, B. 2000. Nitrogen mineralization from fieldapplied beef cattle feedlot manure or compost. Soil Sci. Soc. Am. J. 64:2024-2030.

Eguillor, P. 2004. Análisis de la situación de la agricultura orgánica. Oficina de Estudios y Políticas Agrarias (ODEPA), Santiago, Chile. Disponible en http:// www.odepa.gob.cl Leído el 8 de enero de 2004. 
Frye, W.W., and R.L. Blevins. 1989. Economically sustainable crop production with legume cover crops and conservation tillage. J. Soil Water Cons. 44:5760.

Harris, G.H., and O.B. Hesterman. 1990. Quantifying the nitrogen contribution from alfalfa to soil and two succeeding crops using $\mathrm{N}^{15}$. Agron. J. 82:129-134.

He, Z.L., A.K. Alva, P. Yan, Y.C. Li, D.V. Calvert, P.J. Stoffella, and D.J. Banks. 2000. Nitrogen mineralization and transformation from composts and biosolids during field incubation in a sandy soil. Soil Sci. 165:161-169.

Hoyt, G.D. 1987. Legumes as a green manure in conservation tillage. p. 96-98. In J.F. Powers (ed.) The role of legumes in conservation tillage systems. Soil Conservation Society of America, Ankeny, Iowa, USA.

INE, 1997. VI Censo Nacional Agropecuario. 223 p. Instituto Nacional de Estadísticas (INE), Santiago, Chile.

Ladd, J.N., M. Amato, and J.M. Aodes. 1985. Decomposition of plant material in Australian soil. III. Residual organic and microbial biomass $\mathrm{C}$ and $\mathrm{N}$ from isotope-labelled legume material and soil organic matter, decomposing under field condition. Aust. J. Soil Res. 23:603-611.

McVay, K.A., D.E. Radcliffe, and W.L. Hargrove. 1989. Winter legume effects on soil properties and nitrogen fertilizer requirements. Soil Sci. Soc. Am. J. 53:18561862.

Mengel, K. 1996. Turnover of organic nitrogen in soils and its availability to crops. Plant Soil 181:83-93.

Ormeño, J. 2002. Manejo y control de malezas en viñedos orgánicos. 11 p. In Seminario Actualización en el Conocimiento de las Enfermedades Causadas por Virus en Frutales de Carozo y Vides. Los Andes. Julio de 2002. Instituto de Investigaciones Agropecuarias, Centro Regional de Investigación La Platina, Santiago, Chile.
Ovalle, C., S. Urquiaga, A. del Pozo, E. Zagal, and S. Arredondo. 2006a. Nitrogen fixation in six forage legumes in Mediterranean Central Chile. Acta Agric. Scand. B Plant Soil Science 56(4)277-293.

Ovalle, C., M.I. González, A. del Pozo, J. Hirzel, I. Pino, and S. Urquiaga. 2006b. Contribution and transfer of nitrogen from cover crops to raspberry plant using isotopic techniques with ${ }^{15} \mathrm{~N}$. Acta Hortic. (In press).

Raison, R.J., M.J. Connell, and P.K. Khanna. 1987. Methodology for studying fluxes of soil mineral $\mathrm{N}$ in situ. Soil Biol. Biochem. 19:521-530.

Snoeck, D., F. Zapata, and A. Domennach. 2000. Isotopic evidence of the transfer of nitrogen fixed by legumes to coffee trees. Biotechnol. Agron. Soc. Environ. 4:95100.

Stute, J.K., and J.L. Posner. 1995. Synchrony between legume nitrogen release and corn demand in the upper Midwest. Agron. J. 87:1063-1069.

Sullivan, P. 2003. Overview of cover crop and green manures. 15 p. Technical Publication. Appropriate Technology Transfer for Rural Areas (ATTRA), National Sustainable Agriculture Information, USDA, Fayetteville, Arkansas, USA.

Tisdale, S., W. Nelson, J. Havlin, and J. Beaton. 1999. Soil fertility and fertilizers. An introduction to nutrient management. 503 p. 6th ed. Prentice-Hall, Upper Saddle River, New Jersey, USA.

Varco, J., M. Frye, S. Smith, and J. MacKown. 1993. Tillage effects on legume decomposition and transformation of legume and fertilizer nitrogen-15. Soil Sci. Soc. Am. J. 57:750-756. 